\title{
Field synchronized bidirectional current in confined driven colloids
}

\author{
Fanlong Meng $\odot,,^{1,2, *}$ Antonio Ortiz-Ambriz $\odot,{ }^{3,4, *}$ Helena Massana-Cid $\odot,{ }^{3}$ Andrej Vilfan $\odot, 1,5$ \\ Ramin Golestanian $\odot{ }^{1,6, \dagger}$ and Pietro Tierno $\oplus^{3,4,7, \ddagger}$ \\ ${ }^{1}$ Max Planck Institute for Dynamics and Self-Organization (MPIDS), D-37077 Göttingen, Germany \\ ${ }^{2}$ CAS Key Laboratory for Theoretical Physics, Institute of Theoretical Physics, Chinese Academy of Sciences, Beijing 100190, China \\ ${ }^{3}$ Departament de Física de la Matèria Condensada, Universitat de Barcelona, 08028 Barcelona, Spain \\ ${ }^{4}$ Institut de Nanociència i Nanotecnologia, Universitat de Barcelona, 08028 Barcelona, Spain \\ ${ }^{5}$ J. Stefan Institute, Jamova 39, SI-1000 Ljubljana, Slovenia \\ ${ }^{6}$ Rudolf Peierls Centre for Theoretical Physics, University of Oxford, Oxford OX1 3PU, United Kingdom \\ ${ }^{7}$ Universitat de Barcelona Institute of Complex Systems (UBICS), Universitat de Barcelona, 08028 Barcelona, Spain
}

(Received 5 November 2019; published 23 January 2020)

\begin{abstract}
We investigate the collective colloidal current that emerges when strongly confined magnetic microspheres are subjected to a biased, but spatially uniform, precessing magnetic field. We observe a net bidirectional current composed of colloidal particles which periodically meet assembling into rotating dimers, and exchange their positions in a characteristic, "ceilidh"-like dance. We develop a theoretical model which explains the physics of the observed phenomena as dimer rupture and onset of current, showing agreement with Brownian dynamic simulations. By varying the tilt angle and the frequency of the applied field, we discover two separate transport mechanisms based on different ways the dimers break up during particle transport. Our results demonstrate an effective technique to drive microscale matter by using a combination of confinement and homogeneous field modulations, not based on any gradient of the applied field.
\end{abstract}

DOI: 10.1103/PhysRevResearch.2.012025

Introduction. Strongly confined systems present equilibrium and dynamical properties which significantly differ from those of their bulk counterparts. Examples are widespread across condensed matter physics and range from plasma transport [1] to electronic flow in graphene [2,3], microfluidic $[4,5]$, glassy [6,7], soft [8], and active matter [9,10] systems. A tunable, mesoscopic model system where the effect of confinement can be investigated in real space/time consists of microscopic colloidal particles, where the pair interactions can be tuned in situ by application of an external field [11]. As such, there have been extensive investigations on the equilibrium phases and dynamics that emerge when microscale particles are strongly confined between flat surfaces or pores $[12,13]$. Depending on the type of confinement, the particle distance from the surfaces or the pair interactions, the assembled particles may form novel crystalline phases [14], buckled and frustrated states $[15,16]$, modifying different physical scenarios as packing [17], melting [18,19], and crystallization [20].

The overall dynamics may completely change when considering out-of-equilibrium systems, such as particles driven

\footnotetext{
*These authors contributed equally to this work.

†ramin.golestanian@ds.mpg.de

†ptierno@ub.edu
}

Published by the American Physical Society under the terms of the Creative Commons Attribution 4.0 International license. Further distribution of this work must maintain attribution to the author(s) and the published article's title, journal citation, and DOI. by external fields or forces. On the theoretical side, much progress has been made in understanding the dynamics of confined driven systems both in one [21,22] and two [23,24] dimensions. However, engineering external forces that can actuate the particles uniformly across the sample and produce controlled transport is challenging and therefore experiments are still lagging behind theoretical propositions. In this context, a particularly appealing class of systems are driven magnetic particles, which present emergent collective dynamics $[25,26]$ apart from their potential applications in technological fields such as microfluidics and lab-on-a-chip [27,28]. However, in most cases, the colloidal current is usually produced either via magnetic torque applied to anisotropic particles [29-35], hydrodynamic flows [36-40], or from the presence of external field gradients acting over isotropic spheres [41-43]. Forces resulting from gradients of external fields are usually more difficult to control over spatially extended surfaces or to be switched on/off quickly on command.

In this Rapid Communication, we show that a combination of confinement and dipolar interactions induced by a spatially uniform magnetic modulation can be used in tandem to generate a large-scale, field synchronized colloidal current of isotropic magnetic particles. Recently, we reported that this strongly confined system presents a rich state diagram characterized by synchronous and asynchronous rotating dimers, and random exchange processes with no current [44]. Here, we use an external, biased precessing field to dynamically assemble the particles into transient rotating dimeric states that periodically break and reform, producing a net bidirectional current. We develop a theoretical model which unveils two different mechanisms of motion underlying the colloidal current 
observed in the experiments. Our model is complemented by Brownian dynamics simulations of a large-scale system.

Experimental system. We use monodisperse paramagnetic colloidal particles with diameter $d=2.8 \mu \mathrm{m}$ and magnetic volume susceptibility $\chi \sim 0.4$ (Dynabeads M-270, Invitrogen). These particles exhibit paramagnetic properties since they are composed of a polymer matrix evenly doped with nanoscale ferrite grains. The particles are dispersed in Millipore water (Milli-Q) and strongly confined within a thin cell of thickness $h<2 d$, where $h \in[2.8-5.6 \mu \mathrm{m}]$. Such a cell is created by sandwiching the particle suspension between a microscope slide (thickness $0.96-1.06 \mathrm{~mm}$, Corning) and a coverslip (No. 1, Thermo Scientific Menzel). The particle dynamics are visualized via an upright optical microscope (Eclipse Ni, Nikon) equipped with a charge-coupled-device camera (Scout scA640-74f, Basler). We generate a timedependent magnetic field by using a set of three, custommade, perpendicular magnetic coils, two of which are connected to a power amplifier (DAP Palladium P-400, Vintage) controlled via a wave-form generator (TGA1244, TTi). A static field is obtained by a third coil connected to a DC power supply (EL302RT, TTi).

Once in the confining cell, the particles sediment to the bottom surface, where they display thermal fluctuations close to the bottom plane $(x, y)$. We create a bidirectional edge current by applying a biased precessing magnetic field $\boldsymbol{B}(t)=B_{0}\left\{\left[\sin (\theta) \cos \left(\phi_{B}\right)+\cos (\theta) \sin (\delta)\right] \boldsymbol{x}+\sin (\theta)\right.$ $\left.\sin \left(\phi_{B}\right) y+\cos (\theta) \cos (\delta) z\right\}$, where $\phi_{B}=\omega t$ is the phase of the field, $\omega=2 \pi f$ the angular frequency, $B_{0}$ the amplitude, and $\theta$ the precession angle that we keep constant at $26.9^{\circ}$. As shown in Fig. 1(c), this field performs a conical precession around the vertical $(z)$ axis, but its rotation around $z$ is slightly shifted by a tilt angle $\delta$, which breaks the rotational symmetry of the system. The effect of such field is illustrated in Fig. 1(a): It first produces a buckled state composed of "up" and "down" particles close to the top and bottom surface, respectively. When the induced magnetic dipolar interactions change from repulsive to attractive during one field cycle, the particles transiently assemble into rotating dimers, which then break apart. The result is a field synchronized bidirectional current where up and down particles translate with opposite, frequency tunable, velocities $v_{\uparrow, \downarrow} \propto \pm d f / \sqrt{\Phi}$ [Figs. 1(d) and 1(e)]. Here, $\Phi=N \pi d^{2} /(4 A)$ is the normalized area fraction, $N$ the number of particles, and $A$ the observation area. We observe this current only when $\delta \neq 0$, while for a simple precessing field the dimers may randomly exchange particles across the sample but with zero average current. We find that the current arises already for very small values of $\delta$, which are able to break the spatial symmetry of the precessing field. However, the precise value of $\delta$ is difficult to determine experimentally, thus we mainly explore these parameters using numerical simulations (see below). Moreover, our experimental system displays dislocations which could reduce the average particle currents. These lattice defects may arise from thermal fluctuations or from disorder present in the system in the form of particle polydispersity or surface asperities.

Theory. To understand the colloidal current, we have developed a theoretical model which considers the effect of confinement and magnetic dipolar interactions. We start our analysis with the dynamics of a single-particle pair (dimer),

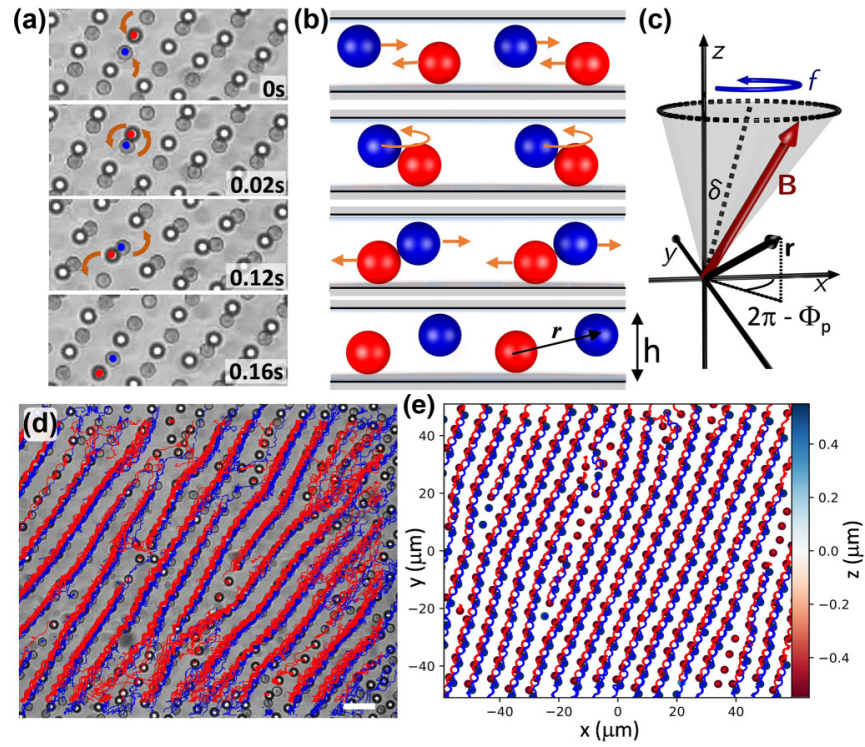

FIG. 1. (a) Optical microscope images showing the exchange process between particles confined within a cell of thickness $h=$ $4 \mu \mathrm{m}$ and located close to the upper (dark) and lower (bright) plane. The applied precessing field has $B_{0}=7.2 \mathrm{mT}, \theta=26.9^{\circ}$, and $f=$ $6 \mathrm{~Hz}$. (b) Sequence of schematics corresponding to (a) and showing the exchanged-based transport mechanism. (c) Sketch of the biased precessing magnetic field with tilt angle $\delta$. (d) Experiments and (e) corresponding numerical simulations of a large system driven by the same field as (a) with $\delta \sim 7^{\circ}$. The particle trajectories are superimposed to the image in blue (dark) and red (bright) for up and down particles. Scale bar is $10 \mu \mathrm{m}$. See corresponding Video 1 in the Supplemental Material [45].

neglecting magnetic interactions with all other particles in the system. In particular, we investigate the stability of a dimer in a precessing magnetic field, as the rupture of a dimer is a precondition for the exchange process and the emergence of a current. When subjected to an external field $\boldsymbol{B}$, an individual paramagnetic colloidal particle acquires a dipole moment $\boldsymbol{m}=\pi d^{3} \chi \boldsymbol{B} /\left(6 \mu_{0}\right)$, with $\mu_{0}$ denoting the permeability of vacuum. The biased precessing field can be decomposed in a static part $\boldsymbol{B}_{1}=B_{0}(\cos \theta \sin \delta, 0, \cos \theta \cos \delta)$, and a dynamic one $\boldsymbol{B}_{2}=B_{0}\left(\cos \phi_{B} \sin \theta, \sin \phi_{B} \sin \theta, 0\right)$. Let us consider a dimer consisting of two particles $(1,2)$, with separation distance $\boldsymbol{r}=\boldsymbol{r}_{2}-\boldsymbol{r}_{1}$ where $\boldsymbol{r}_{1}=-1 / 2\left(\rho \cos \phi_{p}, \rho \sin \phi_{p}, z\right)$, and $\boldsymbol{r}_{2}=1 / 2\left(\rho \cos \phi_{p}, \rho \sin \phi_{p}, z\right)$. Here, $\rho$ is the horizontal component of the center to center distance and $\phi_{p}$ its angle with the $\boldsymbol{x}$ axis. The dipolar interaction between the pair is given by

$$
U_{\mathrm{dip}}=-\frac{\mu_{0}}{4 \pi r^{5}}\left[3\left(\boldsymbol{m}_{1} \cdot \boldsymbol{r}\right)\left(\boldsymbol{m}_{2} \cdot \boldsymbol{r}\right)-\left(\boldsymbol{m}_{1} \cdot \boldsymbol{m}_{2}\right) \boldsymbol{r}^{2}\right],
$$

with force $F_{\rho}=-\partial U_{\text {dip }} / \partial \rho$. The dynamics of the pair can be described by the coupled equations

$$
\begin{aligned}
\dot{\rho} & =\frac{2}{\zeta} F_{\rho}, \\
\dot{z} & =-\frac{2}{\zeta} \frac{\partial U_{\mathrm{dip}}}{\partial z}, \\
\dot{\phi}_{p} & =-\frac{2}{\rho^{2} \zeta} \frac{\partial U_{\mathrm{dip}}}{\partial \phi_{p}},
\end{aligned}
$$



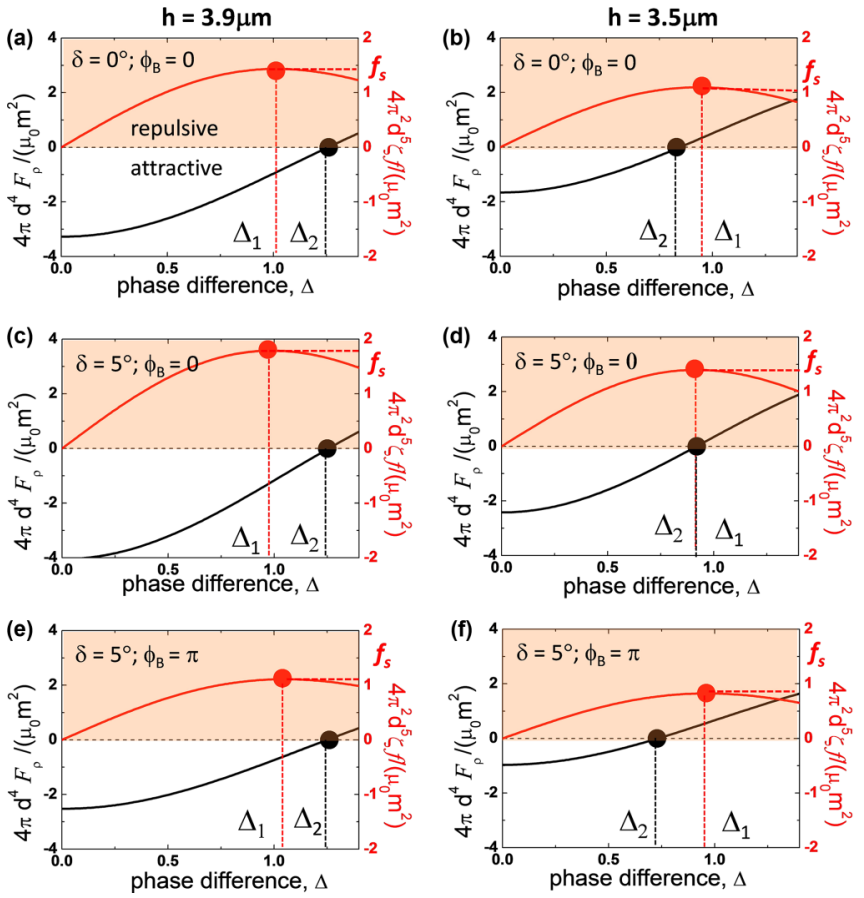

FIG. 2. (a)-(f) Normalized dipolar force $F_{\rho}$ (black curves) and driving frequency $f$ (red curves) vs the phase-lag difference $\Delta=$ $\phi_{B}-\phi_{p}$ for a pair of paramagnetic colloids. The left column [(a), (c), (e)] refers to a system with thickness $h=3.9 \mu \mathrm{m}$, and illustrates the breakage of a dimer after asynchronous slippage (mechanism M1). The right column [(b), (d), (f)] refers to a thickness $h=3.5 \mu \mathrm{m}$ and corresponds to the direct dimer breaking (M2). The first row [(a), (b)] refers to the case of $\delta=0$ and $\phi_{B}=0$, and the middle and bottom rows to $\delta=5^{\circ}, \phi_{B}=0[(\mathrm{c}),(\mathrm{d})]$ and $\phi_{B}=\pi[(\mathrm{e}),(\mathrm{f})]$.

where $\zeta$ is the drag coefficient. We analyze Eqs. (2) for two cases: $\delta=0$ and $\delta \neq 0$.

(a) When $\delta=0$, Eq. (1) can be simplified as

$$
\frac{U_{\mathrm{dip}}}{\mu_{0} m^{2}}=\frac{\rho^{2}+z^{2}-3\left[\rho \sin \theta \cos \left(\phi_{p}-\phi_{B}\right)+z \cos \theta\right]^{2}}{4 \pi\left(\rho^{2}+z^{2}\right)^{5 / 2}},
$$

which is minimal when the dimer is oriented in the direction of the field, $\phi_{p}=\phi_{B}$. In a static vertical magnetic field $\boldsymbol{B}=\boldsymbol{B}_{1}$, the two particles will form a stable dimer if $F_{\rho}<0$, which is the case when $h>d(1+1 / \sqrt{5})$. Then the separation is fixed to $\rho=\sqrt{2 h d-h^{2}}$ and $z=h-d$. If the magnetic field is dynamic $\boldsymbol{B}=\boldsymbol{B}_{1}+\boldsymbol{B}_{2}(t)$, we can identify two regimes of motion depending on the field frequency $f$. At low frequencies, the dimer follows the field with a constant phase lag $\Delta=\phi_{B}-\phi_{p}$. Above a threshold frequency $f_{s}$, the magnetic torque becomes insufficient for the dimer to follow the field rotation. The maximum torque is reached at a phase lag $\Delta_{1}$ (Fig. 2). The dimer dynamics becomes asynchronous and because of phase slippage, $\Delta$ grows in a sawtooh-like manner.

The in-plane component of the magnetic dipolar force between the particles is attractive in a range of phase differences $-\Delta_{2}<\Delta<\Delta_{2}$ and repulsive otherwise. For example, this boundary angle is $\Delta_{2}=1.26$ for $h=3.9 \mu \mathrm{m}$ and $\theta=0.15 \pi$ [Fig. 2(a)]. The dimensionless threshold frequency for slippage, defined as $\mathcal{F}=4 \pi^{2} d^{5} \zeta f /\left(\mu_{0} m^{2}\right)$, is $\mathcal{F}_{1}=1.44$. At this $h$, repulsion always occurs after phase slippage, $\Delta_{2}>\Delta_{1}=$
1.01. In other words, a synchronously rotating dimer is always stable. After phase slippage, the phase difference will run through all angles and inevitably reach the repulsive regime. Therefore, the dimer ruptures as a consequence of a phase slip above the threshold frequency $f_{s}$. As we will show later, this rupture pathway will give rise to one transport mechanism, which occurs in thicker cells and which we denote with M1. In contrast, in thinner cells, e.g., $h=3.5 \mu \mathrm{m}$, the dimer rupture can occur before the phase slippage, $0.85=\Delta_{2}<\Delta_{1}=0.94$ [Fig. 2(b)], at a frequency $\mathcal{F}_{2}=1.08$. This different transport mechanism is denoted as M2 and is characterized by a direct rupture from the synchronous state.

When the dimer breaks, it releases the up and down particles along their respective planes. After being expelled from the dimer, the two particles can be attracted by other magnetic particles forming a new dimeric state when the new phase difference $\Delta$ lies in the attractive range specified above. Since for $\delta=0$ the precessing field is spatially symmetric, there will be no preferred direction for the particle exchange, and thus no net current.

(b) When $\delta \neq 0$, there is a preferred spatial direction for the particle exchange and the net current is not zero. Here, we fix $\delta=5^{\circ}$ and again find that the dimer breakage can occur on two distinct pathways, M1 and M2, depending on the thickness $h$ (Fig. 2). We start by considering a larger $h$, i.e., $h=3.9 \mu \mathrm{m}$. As shown in Fig. 2(c), at $\phi_{B}=0$ the maximum torque is reached at a phase lag $\Delta_{1}=0.99$, corresponding to a slippage frequency $\mathcal{F}_{1}=1.79$. The calculated slippage frequency depends on the phase of the magnetic field $\phi_{B}$-it is minimal at $\phi_{B}=\pi$, with $\mathcal{F}_{1}(\pi)=1.11$ [Fig. 2(e)]. In the following, we use an adiabatic approximation-we assume that at each phase $\phi_{B}$, the lag angle $\Delta$ quickly reaches a value for which the torque corresponds to that needed for uniform motion with the field frequency.

For all angles $\phi_{B}$, repulsion occurs after phase slippage, $\Delta_{2}>\Delta_{1}$. Therefore, the breakage follows mechanism M1 (it is preceded by phase slippage) and the particle current occurs at the minimal slippage frequency $\mathcal{F}_{1}(\pi)$.

The mechanism of dimer rupture is different for small $h$, e.g., $h=3.5 \mu \mathrm{m}$. As shown in Figs. 2(d) and 2(f), the slippage frequency is $\mathcal{F}_{1}=1.40$ at $\phi_{B}=0$, corresponding to a phase lag $\Delta_{1}=0.92$. The magnetic force between the particles turns from attractive to repulsive at $\Delta_{2}$, which can be smaller than $\Delta_{1}$ for some $\phi_{B}$ angles, meaning that the dimer can directly break in the synchronous regime (mechanism M2). The minimum frequency is $\mathcal{F}_{c}=\mathcal{F}_{2}(\pi)=0.77$ at $\phi_{B}=\pi$ [Fig. 2(f)].

To verify that the single-dimer stability analysis provides a good prediction of the collective current and to further elucidate its dependence on different experimental parameters, we complement the theoretical approach with Brownian dynamics simulations. We modified the free package LAMMPS [46] by including an overdamped integrator and the induced magnetic dipole moments in the particles. Our simulation neglects hydrodynamic interactions since these interactions are screened by the close proximity of the planes. We consider $N$ paramagnetic colloids at positions $\boldsymbol{r}_{i} \equiv\left(x_{i}, y_{i}, z_{i}\right)$. To fix a packing fraction, we set periodic boundary conditions in the $(\boldsymbol{x}, \boldsymbol{y})$ plane, while $z_{i}$ is constrained by placing two hard walls at positions $\pm h / 2$. We integrate the overdamped equations of 

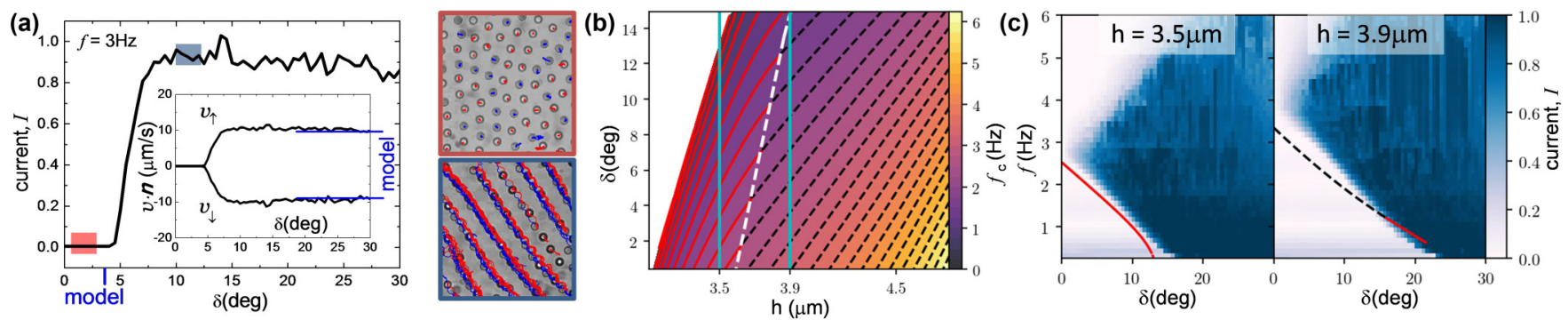

FIG. 3. Normalized current amplitude $I$ vs tilt angle $\delta$ for one driving frequencies. We define $I=2|\boldsymbol{v}| /[f d \sqrt{\pi /(\sqrt{3} \Phi)}]$, where $\Phi$ is the normalized area fraction, and $|\boldsymbol{v}|=\sum\left|\boldsymbol{v}_{i}\right|$ the mean velocity of the particles. The velocity of a particle $i$ at an elevation $z_{i}$ from the middle plane is given by $\boldsymbol{v}_{i}=\left\langle\left(v_{y, i} \boldsymbol{x}-v_{x, i} \boldsymbol{y}\right) z_{i} /\left|z_{i}\right|\right\rangle$. The inset shows the corresponding particle velocities $v$ vs $\delta$ along the average direction of the current, indicated by a director $\boldsymbol{n}$. Experimental images on the right are indicated by the corresponding shaded region in the graph. See corresponding Video 2 in the Supplemental Material [45]. (b) Theory: State diagram $\left(h, \delta, f_{c}\right)$ illustrating the regions related to the two different mechanisms of net colloidal current. (c) Diagrams in the $(\delta, f)$ plane combining the simulation results (continuous color map) and the model (continuous thick line) for two different thickness corresponding to the two mechanisms.

motion,

$$
\gamma \frac{d \boldsymbol{r}_{i}}{d t}=\sum_{j \neq i} \boldsymbol{F}_{\mathrm{int}}\left(\boldsymbol{r}_{i}-\boldsymbol{r}_{j}\right)+\boldsymbol{F}_{w}+\boldsymbol{F}_{g}+\eta(t)
$$

where $\gamma$ is the viscous friction, $\boldsymbol{F}_{\text {int }}\left(\boldsymbol{r}_{i}-\boldsymbol{r}_{j}\right)$ is the total force exerted on particle $i$ by particle $j, \boldsymbol{F}_{w}$ is the normal force exerted by the walls on particle $i$, and $\boldsymbol{F}_{g}$ the gravitational force. The first term can be written as $\boldsymbol{F}_{\text {int }}\left(\boldsymbol{r}_{i}-\boldsymbol{r}_{j}\right)=$ $-\nabla U_{\text {int }}\left(\boldsymbol{r}_{i}-\boldsymbol{r}_{j}\right)$, where the interactions between the particles are given by the dipolar term with the addition of a repulsive Weeks-Chandler-Andersen (WCA) potential $U_{\mathrm{WCA}}$ [44], $U_{\mathrm{int}}\left(\boldsymbol{r}_{i}-\boldsymbol{r}_{j}\right)=U_{\mathrm{dip}}\left(\boldsymbol{r}_{i}-\boldsymbol{r}_{j}\right)+U_{\mathrm{WCA}}\left(\left|\boldsymbol{r}_{i}-\boldsymbol{r}_{j}\right|\right)$. The first one is given by Eq. (1) and in its calculation we take into account the contribution of the magnetic field induced by nearest particles. This is calculated iteratively, and we find that typically only two iterations for the dipole moments were enough to converge. Thus, to speed up the simulations we set up a cutoff for the magnetic interaction of $\sim 30 \mu \mathrm{m}$. The interaction force between particle $i$ and the walls is given by $\boldsymbol{F}_{w}(z)=-\nabla U_{w}(z)$, where $U_{w}(z)$ is also approximated by a WCA potential. The gravitational force is given by $\boldsymbol{F}_{g}=$ $-\Delta \rho V g z$, where $g$ is the gravitational acceleration, $V$ the particle volume, and $\Delta \rho$ is the density mismatch between the particle and water. Finally, $\boldsymbol{\eta}(t) \equiv\left(\eta_{x}, \eta_{y}, \eta_{z}\right)$ in Eq. (4) are random Gaussian variables with mean $\left\langle\eta_{i}(t)\right\rangle=0$ and correlation function $\left\langle\eta_{i}(t) \eta_{j}\left(t^{\prime}\right)\right\rangle \equiv 2 k_{\mathrm{B}} T \gamma \delta_{i j} \delta\left(t-t^{\prime}\right)$, with $T$ being the temperature and $k_{\mathrm{B}}$ the Boltzmann constant [47]. Illustrative videos resulting from the simulation can be found in the Supplemental Material [45] and in this Ref. [48].

Results. In Fig. 3(a) we explore the dependence of the normalized particle current $I$ on the tilt angle $\delta$. We define the current such that $I=1$ corresponds to the fully synchronized transport, where up and down particles form one dimeric state per field cycle (see the caption of Fig. 3). In the simulation and in the experiments, as predicted by the model, we find that when $\delta=0, I=0$, and the dimers simply break or exchange without showing any net current [Fig. 3(a)]. The addition of a small tilt angle $\delta$ to the precessing field is already able to break its spatial symmetry, and to induce the bidirectional current. Indeed we find the threshold value $\delta \sim 5^{\circ}$ for a frequency of $f=3 \mathrm{~Hz}$. Above $\delta \sim 10^{\circ}, I$ reaches a saturation value where almost all particles are mobilized. Since the particles are sliding in opposite directions along parallel planes, their velocities display a bifurcation diagram with two symmetric branches as shown in the inset of Fig. 3(a). The threshold tilt angle and the saturation speed can both be predicted from our model and give, for the same field parameters, similar values of $\delta=3.3^{\circ}$ and $\boldsymbol{v} \cdot \boldsymbol{n}= \pm 11 \mu \mathrm{m} / \mathrm{s}$.

Furthermore, we use the model to calculate a full state diagram in terms of the critical frequency $f_{c}$ as a function of $\delta$ and $h$ [Fig. 3(b)]. The two mechanisms of breakage are represented by continuous red (M1) and dashed black (M2) lines. Their occurrence depends mainly on the thickness of the confining cell. The theoretical results can be directly compared with numerical simulations, as shown in the two cuts through this diagram in Fig. 3(c). These images represent color maps of the current in the $(\delta, f)$ plane for a $h=3.5 \mu \mathrm{m}$ (left) and $h=3.9 \mu \mathrm{m}$ (right) and are overlaid with the corresponding theoretical lines lines in black (M1) and red (M2).

The collective nature of the current implies a strong dependence of the velocity on the particle density, here measured in terms of $\Phi$. Figure 4 shows the current $I$ for different particle concentrations, all measured at a fixed frequency of $f=3 \mathrm{~Hz}$ in a cell of thickness $h=4 \mu \mathrm{m}$. Below $\Phi<0.2$ the particles form a disordered phase composed of dispersed particles and thus the current cannot be generated for any $\delta$. Starting from $\Phi \sim 0.2$ the previously planar particle arrangement buckles and particles assume up and down configurations. The periodic exchange of their positions gives rise to a net current. In this condition, the magnitude of the velocity is given by $|\boldsymbol{v}|=\frac{f d}{2} \sqrt{\frac{\pi}{\Phi \sqrt{3}}}$, and it decreases as the system becomes more packed, i.e., $\Phi$ increases. Above $\Phi \sim 0.4$, the particle pairs can no longer complete half rotations without colliding with neighboring dimers (see the notebook [49]), and $I$ decreases continuously until vanishing for $\Phi \gtrsim 0.65$, when the high density of particles causes clustering, which impedes further propagation and leads to a jammed state. As shown in Video 3 in the Supplemental Material [45], such a state is characterized by vibrating up and down particles that are caged by nearest neighbors forming a characteristic superstructure, where the up particles form a triangular lattice, superimposed to a kagome network of down particles. We note that such structure, however, does not represent the ground state of the 


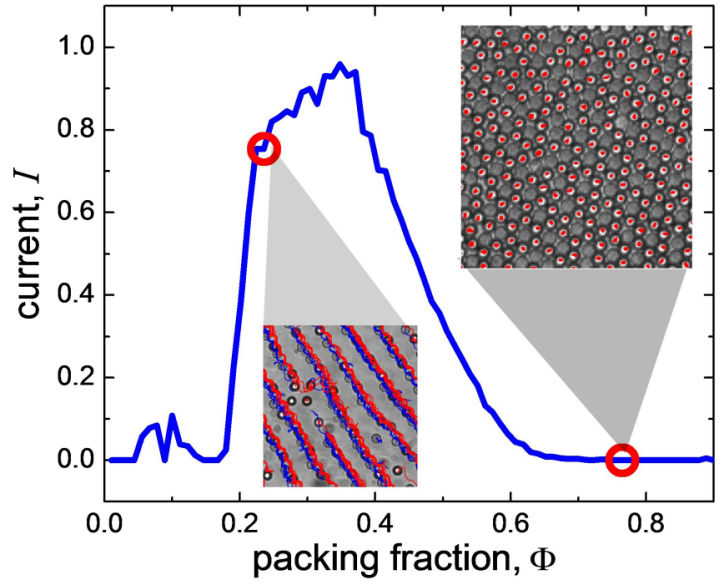

FIG. 4. Colloidal current $I$ vs normalized area fraction $\Phi$ obtained from numerical simulation for a cell with $h=4 \mu \mathrm{m}, B_{0}=$ $7.2 \mathrm{mT}, f=3 \mathrm{~Hz}, \delta=7^{\circ}$. The insets show experimental images at $\Phi=0.23$ (left) and $\Phi=0.77$ (right) with superimposed trajectories of up (down) particles in blue (red). See corresponding Video 3 in the Supplemental Material [45].

buckled system, which instead is characterized by parallel stripes $[15,50]$. The composite magnetic lattice can be easily melted by switching off the applied field and allowing thermal fluctuations to relax the particle to the equilibrium position, i.e., close to the bottom. Thus one could conceive an annealing process where the particles are periodically assembled in the buckled phase in order to reach the stripe phases of the buckled lattice.

To conclude, we investigate the nonequilibrium transport of strongly confined colloidal matter subjected to a biased, homogeneous magnetic modulation. We identify two different mechanisms of motion leading to a bidirectional current where "up" and "down" particles periodically exchange position in a characteristic "ceilidh"-like motion. Such transport mechanisms represent another finding in the field of magnetic active systems, where it is essential to engineer different forms of controlled transport in order to realize cooperative tasks at the microscale. Finally, the main limitation of the current system is related to the cell thickness, since it requires the system to be quasi-two-dimensional $(d<h<2 d)$. Experimentally, the particle current was observed for thicknesses $h \in[3.5,4.5] \mu \mathrm{m}$ and frequencies up to $\sim 40 \mathrm{~Hz}$. However, this does not exclude the possibility to extend our findings to other polarizable objects subjected to time-dependent external fields.

Acknowledgments. F.M. acknowledges the Alexander von Humboldt Foundation for the support. A.O.-A., H.M.-C., and P.T. acknowledge support from the ERC Grant (No. 811234). A.V. acknowledges support from the Slovenian Research Agency (Grant No. P1-0099). P.T. acknowledges support from from MINECO (FIS2016-78507-C2, ERC2018-092827), DURSI (2017SGR1061), and Generalitat de Catalunya under Program "ICREA Acadèmia."
[1] F. L. Hinton and R. D. Hazeltine, Rev. Mod. Phys. 48, 239 (1976).

[2] H. B. Heersche, P. Jarillo-Herrero, J. B. Oostinga, L. M. K. Vandersypen, and A. F. Morpurgo, Nature (London) 446, 56 (2007).

[3] A. H. Castro Neto, F. Guinea, N. M. R. Peres, K. S. Novoselov, and A. K. Geim, Rev. Mod. Phys. 81, 109 (2009).

[4] T. M. Squires and S. R. Quake, Rev. Mod. Phys. 77, 977 (2005).

[5] A. Siria, P. Poncharal, A.-L. Biance, R. Fulcrand, X. Blase, S. Purcell, and L. Bocquet, Nature (London) 494, 455 (2013).

[6] E. Flenner and G. Szamel, Nat. Commun. 6, 7392 (2015).

[7] S. Vivek, C. P. Kelleher, P. M. Chaikin, and E. R. Weeks, Proc. Natl. Acad. Sci. USA 114, 1850 (2017).

[8] S. Perkin and J. Klein, Soft Matter 9, 10438 (2013).

[9] M. C. Marchetti, J. F. Joanny, S. Ramaswamy, T. B. Liverpool, J. Prost, M. Rao, and R. A. Simha, Rev. Mod. Phys. 85, 1143 (2013).

[10] C. Bechinger, R. Di Leonardo, H. Löwen, C. Reichhardt, G. Volpe, and G. Volpe, Rev. Mod. Phys. 88, 045006 (2016).

[11] A. Yethiraj and A. van Blaaderen, Nature (London) 421, 513 (2003).

[12] P. S. Burada, P. Hänggi, F. Marchesoni, G. G. Schmid, and P. P. Talkner, Chem. Phys. Chem. 10, 45 (2009).

[13] H. Löwen, J. Phys.: Condens. Matter 21, 474203 (2009).

[14] N. Osterman, D. Babič, I. Poberaj, J. Dobnikar, and P. Ziherl, Phys. Rev. Lett. 99, 248301 (2007).

[15] Y. Han, Y. Shokef, A. M. Alsayed, P. Yunker, T. C. Lubensky, and A. G. Yodh, Nature (London) 456, 898 (2008).
[16] J. Loehr, A. Ortiz-Ambriz, and P. Tierno, Phys. Rev. Lett. 117, 168001 (2016).

[17] S. Neser, C. Bechinger, P. Leiderer, and T. Palberg, Phys. Rev. Lett. 79, 2348 (1997).

[18] Y. Peng, Z. Wang, A. M. Alsayed, A. G. Yodh, and Y. Han, Phys. Rev. Lett. 104, 205703 (2010).

[19] A. L. Thorneywork, J. L. Abbott, D. G. A. L. Aarts, and R. P. A. Dullens, Phys. Rev. Lett. 118, 158001 (2017).

[20] M. Spannuth and J. C. Conrad, Phys. Rev. Lett. 109, 028301 (2012).

[21] B. Derrida, Phys. Rep. 301, 65 (1998).

[22] D. Lips, A. Ryabov, and P. Maass, Phys. Rev. Lett. 121, 160601 (2018).

[23] P. Hänggi and F. Marchesoni, Rev. Mod. Phys. 81, 387 (2009).

[24] C. Reichhardt and C. J. O. Reichhardt, Rep. Prog. Phys. 80, 026501 (2017).

[25] J. Dobnikar, A. Snezhko, and A. Yethiraj, Soft Matter 9, 3693 (2013).

[26] J. E. Martin and A. Snezhko, Rep. Prog. Phys. 76, 126601 (2013).

[27] N. Pamme, Lab Chip 6, 24 (2006).

[28] P. Tierno, Phys. Chem. Chem. Phys. 16, 23515 (2014).

[29] P. Tierno, R. Golestanian, I. Pagonabarraga, and F. Sagués, Phys. Rev. Lett. 101, 218304 (2008).

[30] F. Y. Ogrin, P. G. Petrov, and C. P. Winlove, Phys. Rev. Lett. 100, 218102 (2008).

[31] A. Ghosh and P. Fischer, Nano Lett. 9, 2243 (2009). 
[32] L. Zhang, J. J. Abbott, L. Dong, K. E. Peyer, B. E. Kratochvil, H. Zhang, C. Bergeles, and B. J. Nelson, Nano Lett. 9, 3663 (2009).

[33] N. Casic, N. Quintero, R. Alvarez-Nodarse, F. G. Mertens, L. Jibuti, W. Zimmermann, and T. M. Fischer, Phys. Rev. Lett. 110, 168302 (2013).

[34] J. Yan, M. Bloom, S. C. Bae, E. Luijten, and S. Granick, Nature (London) 491, 578 (2012).

[35] D. Matsunaga, J. K. Hamilton, F. Meng, N. Bukin, E. L. Martin, F. Y. Ogrin, J. M. Yeomans, and R. Golestanian, Nat. Commun. 10, 4696 (2019).

[36] R. Dreyfus, J. Baudry, M. L. Roper, M. Fermigier, H. A. Stone, and J. Bibette, Nature (London) 437, 862 (2005).

[37] A. Snezhko, M. Belkin, I. S. Aranson, and W.-K. Kwok, Phys. Rev. Lett. 102, 118103 (2009).

[38] C. E. Sing, L. Schmid, M. F. Schneider, T. Franke, and A. Alexander-Katz, Proc. Natl. Acad. Sci. USA 107, 535 (2010).

[39] A. Snezhko and I. S. Aranson, Nat. Mater. 10, 698 (2011).

[40] F. Martinez-Pedrero, A. Ortiz-Ambriz, I. Pagonabarraga, and P. Tierno, Phys. Rev. Lett. 115, 138301 (2015).
[41] B. Lim, V. Reddy, X. Hu, K. Kim, M. Jadhav, R. AbediniNassab, Y. Noh, Y. T. Lim, B. B. Yellen, and C. Kim, Nat. Commun. 5, 3846 (2014).

[42] G. Vieira, T. Henighan, A. Chen, A. J. Hauser, F. Y. Yang, J. J. Chalmers, and R. Sooryakumar, Phys. Rev. Lett. 103, 128101 (2009).

[43] P. Tierno, F. Sagués, T. H. Johansen, and T. M. Fischer, Phys. Chem. Chem. Phys. 11, 9615 (2009).

[44] H. Massana-Cid, A. Ortiz-Ambriz, A. Vilfan, and P. Tierno, arXiv:1911.01698.

[45] See Supplemental Material at http://link.aps.org/supplemental/ 10.1103/PhysRevResearch.2.012025 for three video clips illustrating the experimental and simulation results.

[46] S. Plimton, J. Comput. Phys. 117, 1 (1995).

[47] We numerically integrate Eq. (4) using a finite time step of $\Delta t=10^{-4} \mathrm{~s}$ and use experimental parameters for most quantities.

[48] https://nbviewer.jupyter.org/github/aortiza/Notes/blob/master/ Dimers/ExchangeDetails/Current_Angle.ipynb.

[49] https://nbviewer.jupyter.org/github/aortiza/Notes/blob/master/ Dimers/ExchangeDetails/CurrentVsDensity.ipynb.

[50] F. Leoni and Y. Shokef, Phys. Rev. Lett. 118, 218002 (2017). 